\title{
Medication adherence during laboratory workup for primary aldosteronism: pilot study
}

This article was published in the following Dove Press journal:

Patient Preference and Adherence

Friederike A

Sandbaumhüter ${ }^{1,2}$

Manuel Haschke ${ }^{1,2}$

Bruno Vogt ${ }^{3}$

Jürgen M Bohlender ${ }^{1,2}$

'Institute of Pharmacology,

University of Bern, Bern, Switzerland,

${ }^{2}$ Department of Clinical Pharmacology, Inselspital, Bern University Hospital,

University of Bern, Bern, Switzerland,

${ }^{3}$ Department of Nephrology and

Hypertension, Inselspital, Bern

University Hospital, University of

Bern, Bern, Switzerland
Correspondence: Jürgen M Bohlender Department of Clinical Pharmacology, Inselspital, Bern University Hospital, University of Bern, Freiburgstr 18, 3010 Bern, Switzerland

Tel +4I $3 \mid 6325464$

Fax +4I 3I 6325460

Email juergen.bohlender@insel.ch
Purpose: Current hypertension guidelines stipulate that all incompatible medications be stopped before performing laboratory screening for aldosteronism, but patient adherence is unclear. We measured plasma drug concentrations to determine drug adherence and potential drug bias during biochemical tests.

Patients and methods: Plasma concentrations of 10 antihypertensive drugs were quantified by mass spectrometry in 24 consecutive ambulatory patients with uncontrolled hypertension routinely evaluated for aldosteronism. Drug screening was done before (first visit), and on the day of biochemical tests (second visit) after stopping all incompatible medications. Concentrations above those expected at trough dosing interval defined same-day dose intake.

Results: On the first and second visits, $76 \%$ vs $77 \%$ of prescribed antihypertensive doses could be verified in plasma. A total of $33 \%$ of patients were found to be nonadherent and showed divergent plasma drug results relative to prescriptions ( $21 \%$ drugs not detected $13 \%$ unprescribed drugs found) on first visit, $25 \%$ on the second ( $0 \% / 25 \%$ ), and $46 \%$ for both. A total of $21 \%$ used medication incompatible with the biochemical tests on the second visit. Moreover, $17 \%$ of drug concentrations were below expected trough levels on the first vs $15 \%$ on the second visit. This analysis revealed additional four $(17 \%)$ vs three $(13 \%)$ nonadherent patients who failed same-day dose intake and remained undetected by qualitative drug tests.

Conclusion: Nonadherence was frequent during laboratory evaluations for aldosteronism advocating cautious interpretation of results. A multicenter study is desirable to set the stage for new screening protocols that should incorporate also incentives and checks of drug adherence.

Keywords: adherence, drug, hypertension, screening, aldosterone, spectrometry

\section{Introduction}

Primary aldosteronism (PA) is a frequent and well-known cause of secondary hypertension with an estimated prevalence of $5 \%$ to $10 \%$ among unselected patients with high blood pressure (BP). ${ }^{1}$ Its diagnosis is demanding since plasma renin and aldosterone measurements necessitate standardized laboratory conditions. To avoid biased biochemical results and erroneous conclusions, guidelines stipulate that all drugs with a direct or indirect influence on the renin-angiotensin-aldosterone system (RAAS) should be paused sufficiently in advance before the recommended screening tests can be done. ${ }^{2-4}$ In daily practice, however, patients may not strictly adhere to prescribed drug regimens and the reliability of biochemical test results, therefore, remains unclear. ${ }^{5}$

Adherence to prescribed medication can be verified by pill counts, electronic dispensers, and standardized questionnaires, albeit without final proof of drug intake. Instead, drugs can also be measured directly in plasma by liquid chromatographytandem mass spectrometry (LC-MS/MS), which confirms actual drug use and 
provides drug concentrations for useful pharmacokinetic evaluations. ${ }^{5,6}$ We relied on this novel technology to verify drug adherence during routine laboratory screening for PA and check for potential drug bias of the results.

In a pilot study, we measured plasma drug concentrations in routine patients screened for PA and relied on a neutral study setting to avoid alerting patients as to the study aim. We performed standard qualitative drug screening based on the detection of drugs in plasma and also applied pharmacokinetic concentration thresholds to confirm same-day dose intake. This should improve the detection of nonadherence due to irregular drug use.

\section{Patients and methods}

\section{Clinical protocol and study population}

All new referrals at our hypertension clinic were routinely subjected to our standardized laboratory protocol for secondary hypertension and aldosteronism. On the first visit, clinical routine workup was done, prescribed drugs and doses were recorded, and blood samples for routine biochemistry were obtained between 9:00 am and 5:00 pm. Prescriptions listed in the referral letters were systematically checked for accuracy by interrogating patients, medication cards, the hospital's electronic records system, and caregivers and family members as necessary. All drugs interfering with the RAAS or the sympathetic nervous system and incompatible with plasma renin and aldosterone measurements during PA screening were paused. These included angiotensinconverting enzyme inhibitors, angiotensin or $\beta$-adrenergic receptor blockers, diuretics, and centrally acting sympatholytic drugs. Only amlodipine (aml), lercanidipine (ler), and doxazosin were allowed to treat hypertension thereafter. New treatment plans were established and patients instructed accordingly. A second visit was scheduled 2-4 weeks later to allow for sufficient washout of drug concentrations after discontinuation. Plasma renin and aldosterone concentrations were then measured under standardized conditions. Patients were obliged to take their medication while fasting at 6:30 am. All prescribed and used drugs were checked again for intercurrent changes during the visit. Blood samples for biochemical tests were taken between 8:30 and 9:30 am after 1 hour supine rest.

To study drug adherence, we used first and second visit blood samples from patients who participated in a prospective observational study of new predictive biomarkers while being subjected to this protocol (NCT03034265). The inclusion criteria of this primary study were age $\geq 18$ years, treatment with $\geq 2$ antihypertensive drugs for $\geq 3$ months, and a mean office BP $>140 / 90 \mathrm{mmHg}$ on the first visit (mean of three readings with a semi-automated device). Prespecified exclusion criteria were pregnancy, chronic renal insufficiency grade $\geq 4$, cardiac insufficiency New York Heart Association grade IV, chronic obstructive lung disease grade $\geq 3$, liver insufficiency CHILD grade B or C, drugs significantly interfering with the RAAS or sympathetic nervous activity that could not be paused between the two visits, treatment with aliskiren or a mineralocorticoid receptor antagonist, and a $\mathrm{BP} \geq 180 / 100 \mathrm{mmHg}$ on the first visit. ${ }^{7,8}$ Glomerular filtration rate was estimated using the chronic kidney disease epidemiology collaboration formula. ${ }^{9}$

All 54 new clinic referrals were screened over a 12-month period for participation in the primary study. Twenty-nine patients were eligible, presented no exclusion criteria, and were asked to participate. Of them, 25 patients agreed and were included on the first visit after providing written informed consent. One patient failed to attend the second visit and was excluded. Finally, 24 patients remained for analysis. One screened patient was on spironolactone and excluded whereas none was on aliskiren. Permission for the protocol was obtained from the local ethics board (Ethikkommission des Kantons Bern, BE/20/2016; primary data are with the corresponding author and available upon reasonable request).

\section{Plasma drug assays}

Plasma drug concentrations were quantitated by LC-MS/MS using a Shimadzu (Kyoto, Japan) high-pressure liquid chromatograph coupled to an API 4000 tandem mass spectrometer (AB Sciex, Concord, ON, Canada) and standard operating protocols. ${ }^{10}$ Drug assays were available for aml, ler, nebivolol (neb), metoprolol, bisoprolol, perindoprilat (per), valsartan (val), candesartan, olmesartan, irbesartan (irb), and hydrochlorothiazide. These drugs were assayed in all study patients and all blood samples from both visits under blinded conditions. Corrected peak areas were used for quantitation after internal calibration with deuterated compounds. Calibration curves were generated using test plasma spiked with graded concentrations of the analytes. The lower limit of quantitation (LOQ) was $1.0 \mathrm{ng} / \mathrm{mL}$ except for ler and neb $(0.25 \mathrm{ng} / \mathrm{mL})$, per $(0.5 \mathrm{ng} / \mathrm{mL})$, val and irb $(50 \mathrm{ng} / \mathrm{mL})$. Coefficients of determination $\left(\mathrm{r}^{2}\right)$ were $>0.99$. Within-day test accuracy ranged from $89 \%$ to $111 \%$. Duplicate measurements were performed and mean values were calculated.

For each analyte, expected mean peak $\left(\mathrm{C}_{\max }\right)$ and 24-hour trough concentrations $\left(\mathrm{C}_{\min }\right)$ were extracted from the literature with respect to once daily intake, steady-state conditions, an estimated GFR $>60 \mathrm{~mL} / \mathrm{min}$, and the daily doses used. Ratios of measured $\left(\mathrm{C}_{\mathrm{x}}\right)$ with expected peak concentrations $\left(\mathrm{C}_{\max }\right)$ were calculated for each drug $\left(\mathrm{C}_{\mathrm{x}} / \mathrm{C}_{\max }\right)$. The results were 
given as a precentage. ${ }^{11-23}$ For metoprolol (met), retarded galenics were considered. ${ }^{24}$ Intermediate doses or time points were extrapolated as necessary. A plasma drug test was regarded positive if results were $\geq \mathrm{LOQ}$. Nonadherence was defined as a plasma drug result not consistent with prescriptions. Nonadherence due to missing drug intake (NMI) corresponded to a negative plasma result despite a prescription. Conversely, if a drug was detected in plasma without a prescription, this was regarded as unprescribed drug use or self-medication. Partial NMI was defined, if one or more of prescribed and tested drugs were absent in plasma and complete NMI, if all tests were negative. Moreover, $\mathrm{C}_{\mathrm{x}} / \mathrm{C}_{\max }$ values greater than $\mathrm{C}_{\min } / \mathrm{C}_{\max }$ expected for each drug and dose were accepted as same-day dose intake. Frequencies and percentages with $95 \%$ CIs were calculated, and mean values with SDs.

\section{Results}

Table 1 shows the characteristics of the study population, and the classes and frequencies of prescribed antihypertensives on each visit. Drugs were mostly taken as a single morning dose $(\geq 92 \%)$ and only exceptionally in two divided doses or as a single evening dose. The mean interval between the two visits was $15 \pm 6$ days. On the first visit, all patients except one were on at least one antihypertensive drug classified as incompatible with the laboratory tests for aldosteronism. These drugs were subsequently stopped. On the second visit, no further medication changes were noted in all patients.

On the first visit, 18 different antihypertensive drugs were used vs three on the second and 79 vs 26 individual daily doses were prescribed (Table 2). Of these prescribed doses, $76 \%$ could be verified in plasma on the first vs $77 \%$

Table I Patient characteristics and prescribed medication on the first and second visits (mean $\pm S D$ ); $n=24$

\begin{tabular}{l|l|l}
\hline Age (years) & $54 \pm 13$ & \\
Female (\%) & 42 & \\
Body weight (kg) & $82 \pm 22$ & \\
eGFR (mL/min/I.73 $\left.\mathrm{m}^{2}\right)$ & $81 \pm 24$ & \\
Prescribed medication & First visit & Second visit \\
Number of pills taken, any reason & $5.2 \pm 3.6$ & $3.8 \pm 3.1$ \\
Antihypertensive drugs per patient & $3.2 \pm 1.1$ & $1.1 \pm 0.7$ \\
Number of antihypertensive pills/patient & $2.5 \pm 1.0$ & $1.1 \pm 0.7$ \\
ARB/ACE inhibitors (\%) & 83 & 0 \\
$\beta$-blockers (\%) & 54 & 0 \\
Ca-antagonists (\%) & 88 & 83 \\
Diuretics ${ }^{\mathrm{a}}(\%)$ & 67 & 0 \\
$\alpha$-adrenoceptor blocker (\%) & 21 & 25 \\
Moxonidine (\%) & 8 & 0 \\
\hline
\end{tabular}

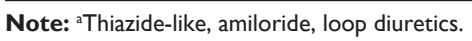

Abbreviations: $A C E$, angiotensin converting enzyme; ARB, angiotensin receptor blocker; eGFR, estimated glomerular filtration rate.

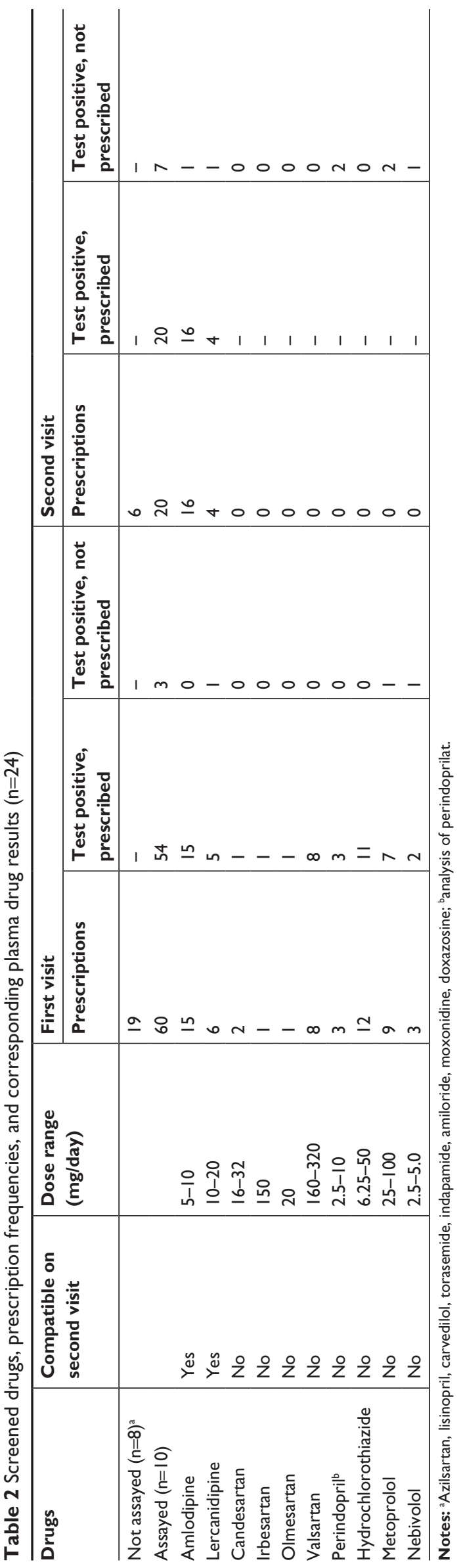


Table 3 Nonadherence and unreported drug use among study participants according to plasma drug screening

\begin{tabular}{|c|c|c|}
\hline Parameter & First visit & Second visit \\
\hline Patients on antihypertensives, $\mathrm{n}(\%)$ & $24(100.0)$ & $18(75.0)$ \\
\hline Nonadherence, $\mathrm{n}(\%, 95 \% \mathrm{Cl})$ & $8(33.3 ; 18.0-53.3)$ & $6(25.0 ; 12.0-44.9)$ \\
\hline Missing intake, $\mathrm{n}(\%, 95 \% \mathrm{Cl})^{\mathrm{a}}$ & $5(20.8 ; 9.2-40.5)$ & $0(0 ; 0-13.8)$ \\
\hline Unprescribed drug use, $\mathrm{n}(\%, 95 \% \mathrm{Cl})^{\mathrm{b}}$ & $3(12.5 ; 3.3-33.5)$ & $6(25.0 ; 12.0-44.9)$ \\
\hline
\end{tabular}

Notes: ${ }^{a} \geq$ I prescription not detected in plasma. ${ }^{b} \geq \mid$ drug detected in plasma without a corresponding prescription.

on the second visit with $90.0 \%$ vs $100 \%$ confirmatory results. Moreover, systematic screening of all plasma samples resulted in three additional positive drug tests on the first and seven on the second visit without a corresponding prescription. All three unexpected findings on the first and six on the second visit concerned drugs classified as incompatible with the planned laboratory tests.

The corresponding rates for nonadherence, missing drug intake, and unreported drug use are shown in Table 3. Nonadherence was detected in $33 \%$ of patients on the first visit, in $25 \%$ on the second visit, and in $46 \%$ for both visits combined. Nonadherence due to missing drug intake was present only on the first visit and partial while unreported drug use was less frequent. On the second (laboratory) visit, unexpected drug use without a prescription was detected in one of four patients. Drugs incompatible with the laboratory tests were used by five (21\%): three (13\%) did not stop these drugs appropriately after the first visit and two (8\%) used such drugs without a prescription.
The plasma drug concentrations are shown in Figure 1. On the first visit, $59.2 \%$ were within $\pm 50 \%$ of $\mathrm{C}_{\max }, 57 \%$ between $\mathrm{C}_{\max }$ and $\mathrm{C}_{\min }$, and $17 \%$ below $\mathrm{C}_{\min }$. On the second visit, the corresponding rates were $78 \%, 35 \%$, and $15 \%$. Compared with qualitative drug screening (Table 3), four patients on the first $(16.7 \%)$ and three on the second visit (12.5\%) showed drug concentrations below $\mathrm{C}_{\min } / \mathrm{C}_{\max }$ and were additionally classified as nonadherent.

\section{Discussion}

As a main result, every second patient presented a mismatch between prescriptions and qualitative plasma drug results on one of the two visits because of nonadherence. More importantly, every fifth patient used incompatible drugs during laboratory tests for PA unexpectedly without a prescription. One reason was failure to stop such drugs beforehand as required. Indeed, the true rates of nonadherence and unreported drug use may have been even higher since a few prescribed drugs could not be verified in plasma.
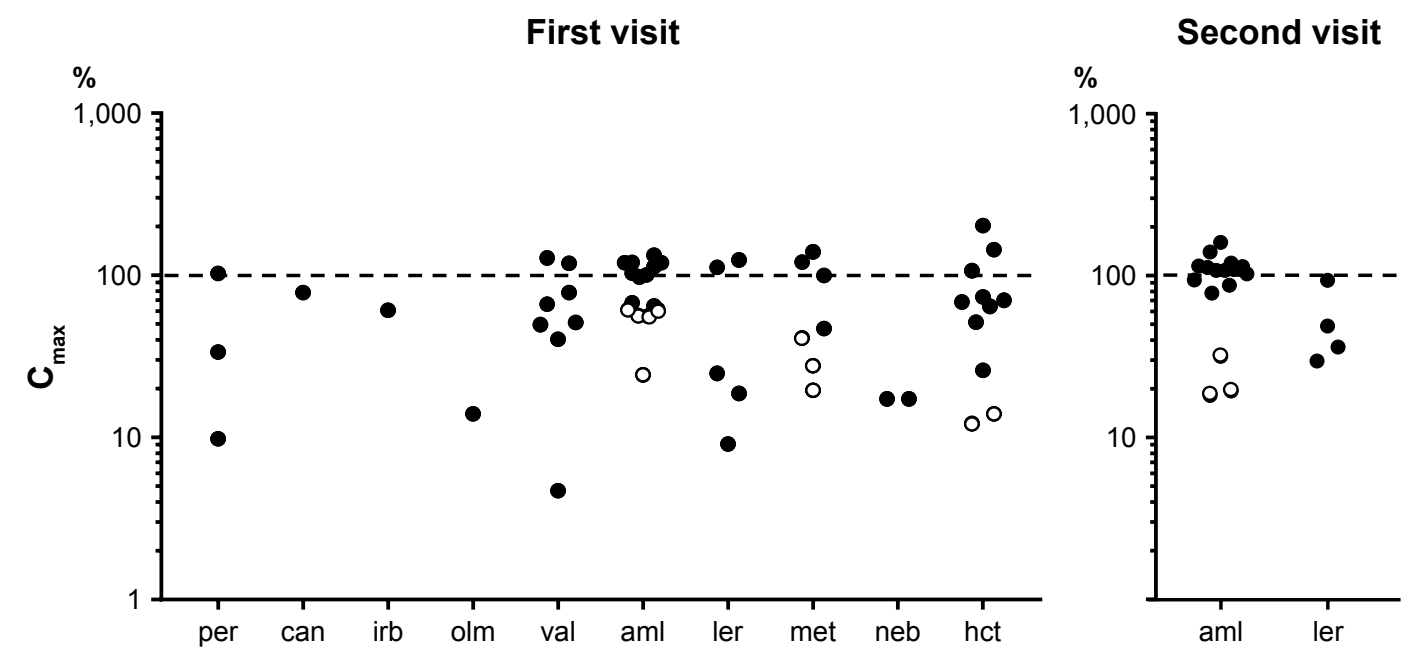

Figure I Plasma concentrations of antihypertensive drugs on the first and second visits expressed as ratios with expected peak concentrations $\left(\mathrm{C}_{\max }\right.$, I00\%); logarithmic representation.

Notes: Only prescriptions confirmed in plasma are shown. Solid circles indicate results $\geq$ expected $\mathrm{C}_{\min }$ and empty circles indicate results $<$ expected $\mathrm{C}_{\text {min }}$. Expected mean $\mathrm{C}_{\max }$ (highest/lowest daily dose) and $\mathrm{C}_{\min }$ values (\% $\mathrm{C}_{\max }$ ) were as follows: per 36.4/15.I ng/mL (7\%/5\%); can $234 \mathrm{ng} / \mathrm{mL}(28 \%) ; \mathrm{irb} \mathrm{2,040} \mathrm{ng/mL} \mathrm{(5 \% );} \mathrm{olm} \mathrm{507} \mathrm{ng/mL} \mathrm{(4 \% );} \mathrm{val}$ $5,550 / 3,940 \mathrm{ng} / \mathrm{mL}$ (18\%/3\%); aml I7.5/I0.5 ng/mL (79\%/57\%); ler 7.7/3.3 ng/mL (5\%/l\%); met 53.5/II.2 ng/mL (70\%/24\%); neb I.5 ng/mL (7\%); hct 260/70 ng/mL (I4\%/9\%). Abbreviations: aml, amlodipine; can, candesartan; hct, hydrochlorothiazide; irb, irbesartan; olm, olmesartan; per, perindopril; val, valsartan; ler, lercanidipine; met, metoprolol; neb, nebivolol. 
Together, our survey suggests that in clinical routine practice, the validity of biochemical PA screening may often be questionable and results unreliable.

Nonadherence to prescribed medication may occur in $10 \%$ to $>50 \%$ of treated hypertensive patients and is usually only partial according to previous study evidence..$^{5,6,25-30}$ We relied on sensitive LC-MS/MS technology for plasma drug screening and found a comparable rate of $21 \%$ on the first study visit. We analyzed blood samples from a biomarker study, which gave us a relatively unbiased picture of drug adherence because it was not advertised as the principal study aim during patient recruitment. A survey of plasma drug levels in hypertensive patients at a US emergency department reported a nonadherence rate of $28 \% .{ }^{27}$ Another study in a French hypertension clinic relied on urinary drug tests and found a much lower nonadherence rate of only $10 \%$. It increased with the number of prescribed drugs per patient and was also associated with a higher BP level. ${ }^{28}$ The patients, however, were on long-term follow-up and their BP was at, or close to, the recommended target values which made frequent medication changes unnecessary. In contrast, our patients were new referrals with often various treatment changes in the past or an irregular follow-up. This readily explains the higher rate of nonadherence during our study.

We also observed a significant rate of unreported drug use or self-medication. Data on self-medication are scarce for hypertensive patients. ${ }^{25-30}$ In one large survey, 38 different drugs were screened in 821 random serum samples by LC-MS/MS and $33 \%$ of positive hits did not correspond to a known prescription. ${ }^{31}$ In another study, $8.3 \%$ of hypertensive patients relied on some kind of self-medication according to plasma drug checks yet without reporting this to the clinic. ${ }^{30}$ Indeed, the current review literature still neglects selfmedication as a relevant topic., ${ }^{5,32}$ Furthermore, adherence questionnaires do not query for potential self-medication and do not help to resolve this problem. ${ }^{6}$

Most unexpected plasma drug findings were $\beta$-adrenoceptor and RAAS blockers, which may hamper the diagnosis of PA by influencing renin and aldosterone secretion and plasma levels. ${ }^{2-4}$ Our survey suggests that two out of ten patients or more may exhibit unreliable biochemical test results in clinical routine practice. Interestingly, current guidelines for the laboratory workup of aldosteronism do not mention nonadherence as a potential source of bias and a caveat. ${ }^{2-4}$ Cautious result interpretations and repeated tests in unclear situations are, therefore, advisable.
We also aimed to improve the detection of nonadherence by analyzing plasma drug concentrations. LC-MS/MS methodology is highly sensitive. Qualitative drug screening based on LOQ thresholds may thus easily generate false positive results when drugs are slowly cleared from plasma and can be detected still days after discontinuation. 5,10,25,26,29,30 Using indexed plasma drug concentrations and expected $\mathrm{C}_{\min }$ as a threshold for same-day drug intake, we additionally revealed up to four nonadherent patients compared with only qualitative plasma drug screening. Drug concentrations in our study were generally around the expected $\mathrm{C}_{\max }$ or within the therapeutic range, that is, between $\mathrm{C}_{\max }$ and $\mathrm{C}_{\min }$. Investigating unselected serum samples, another study found just $40 \%-50 \%$ of drug concentrations within the expected therapeutic limits. ${ }^{31}$ A precise quantitative analysis of drug concentration may be of particular interest whenever drug adherence is critical to clinical outcomes or in research settings.

Some important limitations of our study need to be addressed specifically. As a pilot study in a small number of patients, our survey provided only preliminary results. They require undoubtedly further confirmation by a larger cohort and also in different clinical settings. A few drugs could not be tested in plasma while the true rates of nonadherence may have been possibly higher. Next, we investigated patients without major comorbidity. They probably had also a lower overall pill count than could be expected for the general population and possibly had also a lower propensity to omit medication. ${ }^{28}$ Our inclusion and exclusion criteria were narrow and plasma drug screening was only punctiform. Moreover, there may have been some "white coat effect" that transiently improved drug adherence during laboratory tests compared with the everyday situation. Depending on the clinical situation, nonadherence during laboratory workup for PA may thus be quite variable and difficult to predict. Nevertheless, we believe that our study conclusions remain generally valid despite these confounding factors.

Finally, our study did not address the question of how many patients would possibly be misclassified for PA because of incompatible drugs during the tests for which a larger study with confirmatory PA testing would be necessary. Our protocol fulfilled guideline recommendations and represents current practice standards. We relied on oral instructions, written treatment plans, and a second medication check on the day of the crucial tests, which finally did not suffice to assure medication adherence and valid laboratory conditions in all patients. Additional measures, therefore, 
appear necessary to improve overall drug adherence and concomitantly the reliability of biochemical test results. Educational and motivational checks, controlled drug dispensing, and plasma drug monitoring whenever possible may be useful in this context. ${ }^{5,32}$

\section{Conclusion}

Together, nonadherence to prescriptions was frequent during routine laboratory evaluations for PA. Cautious interpretation of laboratory findings and repeated testing in unclear situations are, therefore, advisable. In addition, extra safety measures, such as plasma drug screening should be considered to minimize the inherent risk of drug bias whenever possible. A larger multicenter survey is desirable to corroborate our findings and set the stage for new screening protocols that should incorporate also incentives and checks of drug adherence.

\section{Acknowledgment}

The study was enabled by a financial grant from the BärSpycher-Stiftung, Switzerland.

\section{Disclosure}

The authors report no conflicts of interest in this work.

\section{References}

1. Rossi GP, Bernini G, Caliumi C, et al; PAPY Study Investigators. A prospective study of the prevalence of primary aldosteronism in 1,125 hypertensive patients. J Am Coll Cardiol. 2006;48(11):2293-2300.

2. Funder JW, Carey RM, Mantero F, et al. The management of primary aldosteronism: case detection, diagnosis, and treatment: an Endocrine Society clinical practice guideline. J Clin Endocrinol Metab. 2016; 101(5):1889-1916.

3. Leung AA, Daskalopoulou SS, Dasgupta K, et al; Hypertension Canada. Hypertension Canada's 2017 Guidelines for diagnosis, risk assessment, prevention, and treatment of hypertension in adults. Can J Cardiol. 2017;33(5):557-576.

4. Douillard C, Houillier P, Nussberger J, Girerd X. SFE/SFHTA/AFCE consensus on primary aldosteronism, part 2: first diagnostic steps. Ann Endocrinol (Paris). 2016;77(3):192-201.

5. Hamdidouche I, Jullien V, Boutouyrie P, Billaud E, Azizi M, Laurent S. Drug adherence in hypertension: from methodological issues to cardiovascular outcomes. J Hypertens. 2017;35(6):1133-1144.

6. Pandey A, Raza F, Velasco A, et al. Comparison of Morisky Medication Adherence Scale with therapeutic drug monitoring in apparent treatment-resistant hypertension. J Am Soc Hypertens. 2015;9(6): 420-426.e2.

7. Stevens PE, Levin A, Bilous RW; Kidney Disease: Improving Global Outcomes Chronic Kidney Disease Guideline Development Work Group Members. Evaluation and management of chronic kidney disease: synopsis of the kidney disease: improving global outcomes 2012 clinical practice guideline. Ann Intern Med. 2013;158(11):825-830.

8. Vogelmeier CF, Criner GJ, Martinez FJ, et al. Global strategy for the diagnosis, management, and prevention of chronic obstructive lung disease 2017 report. GOLD executive summary. Am J Respir Crit Care Med. 2017;195(5):557-582.
9. Levey AS, Stevens LA. Estimating GFR using the CKD Epidemiology Collaboration (CKD-EPI) creatinine equation: more accurate GFR estimates, lower CKD prevalence estimates, and better risk predictions. Am J Kidney Dis. 2010;55(4):622-627.

10. Maurer HH. Multi-analyte procedures for screening for and quantification of drugs in blood, plasma, or serum by liquid chromatographysingle stage or tandem mass spectrometry (LC-MS or LC-MS/MS) relevant to clinical and forensic toxicology. Clin Biochem. 2005;38(4): 310-318.

11. Louis WJ, Workman BS, Conway EL, et al. Single-dose and steady-state pharmacokinetics and pharmacodynamics of perindopril in hypertensive subjects. J Cardiovasc Pharmacol. 1992;20(3):505-511.

12. Medicines Evaluation Board in the Netherlands. Utrecht, NL, 2012. Public assessment report: candesartan cilexetil Lincosa. EU procedure number NL/H/2048/002-004/DC. Available from: https://db.cbg-meb. nl/Pars/h107585.pdf. Accessed July 01, 2018.

13. Meineke I, Feltkamp H, Högemann A, Gundert-Remy U. Pharmacokinetics and pharmacodynamics of candesartan after administration of its pro-drug candesartan cilexetil in patients with mild to moderate essential hypertension-a population analysis. Eur J Clin Pharmacol. 1997;53(3-4):221-228.

14. Marino MR, Langenbacher K, Ford NF, Uderman HD. Pharmacokinetics and pharmacodynamics of irbesartan in healthy subjects. $J$ Clin Pharmacol. 1998;38(3):246-255.

15. Schwocho LR, Masonson HN. Pharmacokinetics of CS-866, a new angiotensin II receptor blocker, in healthy subjects. J Clin Pharmacol. 2001;41(5):515-527.

16. Müller P, Flesch G, de Gasparo M, Gasparini M, Howald H. Pharmacokinetics and pharmacodynamic effects of the angiotensin II antagonist valsartan at steady state in healthy, normotensive subjects. Eur J Clin Pharmacol. 1997;52(6):441-449.

17. ChenCL, Desai-KriegerD, OrtizS, Kerolous M, Wright HM, GhahramaniP. A single-center, open-label, 3-way crossover trial to determine the pharmacokinetic and pharmacodynamic interaction between nebivolol and valsartan in healthy volunteers at steady state. Am J Ther. 2015; 22(5):e130-e140.

18. Stangier J, Su CA. Pharmacokinetics of repeated oral doses of amlodipine and amlodipine plus telmisartan in healthy volunteers. J Clin Pharmacol. 2000;40(12 Pt 1):1347-1354.

19. Bainbridge AD, Herlihy O, Meredith PA, Elliott HL. A comparative assessment of amlodipine and felodipine ER: pharmacokinetic and pharmacodynamic indices. Eur J Clin Pharmacol. 1993;45(5):425-430.

20. Swissmedic, Schweizerisches Heilmittelinstitut, Bern, Switzerland. Public product information: Lercanidipin Sandoz ${ }^{\circledR}$. Available from: www.swissmedicinfo.ch. Accessed July 01, 2018.

21. Barchielli M, Dolfini E, Farina P, et al. Clinical Pharmacokinetics of Lercanidipine. J Cardiovasc Pharmacol. 1997;29(Suppl 2):S1-S15.

22. Kamali F, Howes A, Thomas SH, Ford GA, Snoeck E. A pharmacokinetic and pharmacodynamic interaction study between nebivolol and the H2-receptor antagonists cimetidine and ranitidine. $\mathrm{Br} J \mathrm{Clin}$ Pharmacol. 1997;43(2):201-204.

23. Welling PG. Pharmacokinetics of the thiazide diuretics. Biopharm Drug Dispos. 1986;7(6):501-535.

24. Kendall MJ. Metoprolol-controlled release, zero order kinetics. J Clin Pharm Ther. 1989;14(3):159-179.

25. Strauch B, Petrák O, Zelinka T, et al. Precise assessment of noncompliance with the antihypertensive therapy in patients with resistant hypertension using toxicological serum analysis. J Hypertens. 2013; 31(12):2455-2461.

26. McNaughton CD, Brown NJ, Rothman RL, et al. Systolic blood pressure and biochemical assessment of adherence: a cross-sectional analysis in the emergency department. Hypertension. 2017;70(2):307-314.

27. Hamdidouche I, Jullien V, Boutouyrie P, Billaud E, Azizi M, Laurent S. Routine urinary detection of antihypertensive drugs for systematic evaluation of adherence to treatment in hypertensive patients. J Hypertens. 2017;35(9):1891-1898. 
28. Jung O, Gechter JL, Wunder C, et al. Resistant hypertension? Assessment of adherence by toxicological urine analysis. J Hypertens. 2013; 31(4):766-774.

29. Gupta P, Patel P, Štrauch B, et al. Biochemical screening for nonadherence is associated with blood pressure reduction and improvement in adherence. Hypertension. 2017;70(5):1042-1048.

30. Ceral J, Habrdova V, Vorisek V, Bima M, Pelouch R, Solar M. Difficult-to-control arterial hypertension or uncooperative patients? The assessment of serum antihypertensive drug levels to differentiate non-responsiveness from non-adherence to recommended therapy. Hypertens Res. 2011;34(1):87-90.
31. Ryan TP, Morrison RD, Sutherland JJ, et al. Medication adherence, medical record accuracy, and medication exposure in real-world patients using comprehensive medication monitoring. PLoS One. 2017; 12(9):e0185471.

32. Burnier M. Drug adherence in hypertension. Pharmacol Res. 2017; 125(Pt B):142-149.

\section{Publish your work in this journal}

Patient Preference and Adherence is an international, peer-reviewed, open access journal that focuses on the growing importance of patient preference and adherence throughout the therapeutic continuum. Patient satisfaction, acceptability, quality of life, compliance, persistence and their role in developing new therapeutic modalities and compounds to optimize clinical outcomes for existing disease states are major areas of interest for the journal. This journal has been accepted for indexing on PubMed Central. The manuscript management system is completely online and includes a very quick and fair peer-review system, which is all easy to use. Visit http://www. dovepress.com/testimonials.php to read real quotes from published authors.

Submit your manuscript here: http://www.dovepress.com/patient-preference-and-adherence-journal 